\title{
Comparação do equilíbrio corporal de mulheres a partir da meia-idade obesas e não-obesas
}

\author{
Body balance comparison between obese and non-obese women from middle-age on
}

\author{
Cristina Oliveira Francisco ${ }^{1}$, Vanessa Takakura Okada ${ }^{1}$, Natalia Aquaroni Ricci ${ }^{2}$,
} Benedito Galvão Benze ${ }^{3}$, José Rubens Rebelatto ${ }^{4}$, Ana Cláudia Garcia de Oliveira Duarte

Estudo desenvolvido no Depto. de Fisioterapia da UFSCar Universidade Federal de São Carlos, São Carlos, SP, Brasil

1 Fisioterapeutas

2 Fisioterapeuta Ms.; Profa. substituta do Depto. de Fisioterapia da UFSCar

3 Matemático; Prof. Dr. do Depto. de Estatística da UFSCar

4 Prof. Dr. do Depto. de Fisioterapia da UFSCar

5 Profa. Dra. do Depto. de Educação Física da UFSCar

ENDEREÇO PARA CORRESPONDÊNCIA

Cristina O. Francisco R. Iwagiro Toyama 191 apto 2 Jd. Paulistano 13564-380 São Carlos SP e-mail: cristinaft05@gmail.com

\section{APRESENTAÇÃO}

maio 2009

ACEITO PARA PUBLICAÇÃO out. 2009
Resumo: Este é um estudo comparativo do efeito da obesidade no equilíbrio estático e dinâmico de mulheres a partir da meia-idade. A amostra foi composta por mulheres acima de 50 anos $(n=80)$, distribuídas segundo o índice de massa corporal em grupo não-obeso $(n=45)$ e obeso $(n=35)$, com médias de idade equivalentes. Foram avaliadas quanto à gordura corporal por bioimpedância e quanto ao equilíbrio pelos testes de apoio unipodal (TAU) e de velocidade máxima de andar (VMA). Os dados foram tratados estatisticamente. No TAU em ambos os membros inferiores o grupo não-obeso permaneceu por mais tempo na posição - 25,6 segundos (s) no membro direito e 24,9 s no esquerdo - do que o grupo obeso (19,0 s no direito e $17,5$ s no esquerdo, $p<0,01)$. No VMA o grupo obeso apresentou marcha mais lenta e velocidade média menor quando comparado ao grupo não obeso $(p<0,027)$. No grupo não-obeso, verificaram-se correlações moderadas entre a gordura corporal e o equilíbrio estático e dinâmico; no grupo obeso não foram encontradas correlações entre a a gordura corporal e a maioria das variáveis de equilíbrio. Os resultados evidenciam que a obesidade contribui para o pior desempenho no equilíbrio de mulheres de meia-idade e idosas.

Descritores: Equilíbrio postural; Envelhecimento; Mulheres; Obesidade

ABSTRACT: This is a comparative study on the effect of obesity on static and dynamic balance in middle-aged and elderly women. The sample was composed by 80 women over 50 years old, distributed according to the body mass index into a nonobese group $(n=45)$ and an obese group $(n=35)$, with similar mean age. Participants were assessed as to body fat by bioimpedance and submitted to the one leg stance (OLS) and maximum walking speed (MWS) tests. Data were statistically analysed. At the OLS on both feet the non-obese group remained longer in position - 25.6 seconds (s) on the right limb and 24,9 s on the left one - than the obese group (19.0 $\mathrm{s}$ on the right, $17.5 \mathrm{~s}$ on the left limb, $p<0.01$ ). At he MWS test the obese group showed slower walking and lower mean speed than the non-obese group $(p<0,027)$. Within non-obese group results, moderate correlations were found between body fat and static and dynamic balance; in the obese group practically no corresponding correlations were found. Results show that obesity contributes to worse balance performance in middle-age and elderly women.

KEY words: Aging; Obesity; Postural balance; Women 


\section{INTRODUÇÃO}

A obesidade pode ser considerada, atualmente, um dos maiores problemas de saúde pública em todo o mundo. Isso se deve ao fato de o excesso de peso e gordura corporal estarem associados ao maior risco de desenvolver doenças como diabetes tipo II, hipertensão arterial, arterosclerose, sobrecarga articular, aumento do colesterol e triglicérides ${ }^{1}$. Além disso, ao modificar a composição estrutural do corpo, a obesidade altera a capacidade funcional do indivíduo, gerando declínio físico, dificuldade na marcha e redução da independência ${ }^{2}$.

A Organização Mundial da Saúde (OMS) define obesidade como uma acumulação de gordura anormal ou excessiva que ocasiona prejuízos à saúde $^{3}$. A prevalência da obesidade é semelhante entre os sexos e o pico de ocorrência está na faixa entre os 45 e 64 anos $^{4}$. Entre os idosos, a obesidade está associada à inabilidade física e incapacidade, sendo mais prevalente nas mulheres ${ }^{2,4}$. As doenças crônicas tornam-se mais freqüentes com o avançar da idade e, nesse caso, a obesidade pode ser fator potencializador para complicações clínicas ${ }^{4}$. Entre as modificações ocasionadas tanto pelo envelhecimento como pela obesidade destaca-se a deterioração do sistema de controle postural. O declínio no equilíbrio e marcha está associado às altas taxas de quedas entre os idosos, sendo esta uma das causas principais de morbidade e mortalidade nessa população ${ }^{2,5,6}$.

O sistema de controle postural envolve a recepção e integração de estímulos sensoriais, o planejamento e a execução de movimentos para controlar o centro de gravidade sobre a base de suporte b $^{7,8}$. A obesidade ocasiona desvantagens mecânicas como o aumento da massa corporal, alterando os momentos de inércia e modificando a expressão do movimento. Esses fatores impõem uma nova situação ao sistema nervoso central, gerando maior dificuldade nas atividades locomotoras ${ }^{10}$.

Indivíduos obesos apresentam alterações metabólicas que levam à redução relativa de força e ao prejuízo da função neuromuscular. Esses fatores, associados à maior concentração de massa na região abdominal, afetam negativamente a manutenção do equilíbrio ${ }^{11,12}$. Estudos mostram associação entre maior índice de massa corporal (IMC) e baixo desempenho em testes de equilíbrio ${ }^{12,13}$. Crianças obesas apresentaram maior comprometimento do equilíbrio, com maior dependência visual para manutenção da estabilidade postural do que crianças de peso normal ${ }^{14,15}$. Outros estudos revelaram risco elevado de quedas entre adultos obesos devido à estabilidade corporal inadequada ${ }^{11,16-18}$.

Quedas e desequilíbrios foram associados à redução da qualidade de vida em adultos obesos ${ }^{16}$. Essas alterações decorrentes da obesidade podem ser prevenidas e tratadas em centros de reabilitação. Contudo, torna-se necessário elucidá-las a fim de melhor direcionar a intervenção à população obesa. O objetivo deste estudo foi comparar o equilíbrio estático e dinâmico de mulheres obesas e não-obesas com idade igual ou superior a 50 anos, a fim de verificar o impacto da obesidade no sistema de controle postural.

\section{METODOLOGIA}

Este é um estudo analítico comparativo sobre o efeito da obesidade no equilíbrio de mulheres a partir da meia-idade. Foi conduzido de acordo com os padrões éticos exigidos e aprovado pelo Comitê de Ética em Pesquisa da UFSCar - Universidade Federal de São Carlos, tendo as participantes assinado o devido termo de consentimento.

Foi selecionada amostra de conveniência dentre 176 mulheres participantes do Programa de Revitalização de Adultos, mantido pela UFSCar em parceria com a Prefeitura Municipal de São Carlos, que busca implementar a assistência ao idoso por meio da manutenção da capacidade física e sociabilização.

Para comparar os efeitos da obesidade no equilíbrio foram formados grupos conforme o estado nutricional, avaliado pelo IMC. Este é um dos indicadores antropométricos mais utilizados na identificação de indivíduos em risco nutricional devido a sua facilidade de aplicação, baixo custo e confiabilidade intra e interavaliadores ${ }^{19,20}$. Para o cál- culo do IMC utilizou-se a fórmula Peso $(\mathrm{kg}) /$ Estatura $\left(\mathrm{m}^{2}\right)^{21}$. O peso e altura foram medidos em balança antropométrica com estadiômetro (Filizola). Foram adotados os pontos de corte recomendados pela $\mathrm{OMS}^{3}$ para a população adulta e idosa: baixo peso (IMC $\left.<18,5 \mathrm{~kg} / \mathrm{m}^{2}\right)$; adequado $\left(18,5 \mathrm{~kg} / \mathrm{m}^{2}=\mathrm{IMC}<25,0 \mathrm{~kg} / \mathrm{m}^{2}\right)$; sobrepeso $\left(25,0 \mathrm{~kg} / \mathrm{m}^{2}=\mathrm{IMC}<30,0 \mathrm{~kg} /\right.$ $\left.\mathrm{m}^{2}\right)$, obesidade $\left(30,0 \mathrm{~kg} / \mathrm{m}^{2}=\mathrm{IMC}<40,0\right.$ $\mathrm{kg} / \mathrm{m}^{2}$ ); e obesidade mórbida (IMC $\geq 40,0$ $\mathrm{kg} / \mathrm{m}^{2}$ ). A divisão dos grupos foi feita pelo corte do IMC $\mathrm{IM}^{21}$ : não-obesas, entre 18,5 $\mathrm{kg} / \mathrm{m}^{2}$ e $25 \mathrm{~kg} / \mathrm{m}^{2}$; e obesas, IMC entre $30 \mathrm{~kg} / \mathrm{m}^{2}$ e $40 \mathrm{~kg} / \mathrm{m}^{2}$.

Os critérios de inclusão no estudo foram: idade igual e superior a 50 anos, sexo feminino e aptidão física para participar do programa de revitalização. Foram excluídas as participantes que apresentavam doenças que impedissem a execução dos testes de equilíbrio, que usassem marca-passo ou próteses metálicas, bem como aquelas com IMC indicativo de baixo peso, sobrepeso ou obesidade mórbida, sendo finalmente selecionadas 80 mulheres.

Para complementar o estudo nutricional foi medida a bioimpedância manual utilizando aparelho eletrônico portátil (Fitness Monitor). Esta é uma alternativa para o cálculo da estimativa de gordura corporal, sendo uma técnica segura, simples, rápida, de alta reprodutibilidade, aplicável à prática clínica e a estudos de campo $^{22-24}$. Inicialmente o aparelho deve ser calibrado com o registro da altura, peso e idade (limite máximo de 80 anos) da participante. Com a participante em bipedestação segurando o apareIho com os braços estendidos na altura dos ombros, é feito o cálculo da bioimpedância, por meio de corrente elétrica, que estima a quantidade de gordura corporal em quilos e em porcentagem. Por se tratar de uma corrente elétrica, no momento da avaliação, não era permitido o uso de objetos metálicos pela participante.

Para a coleta de dados, as voluntárias foram primeiramente contatadas por telefone para o agendamento da avaliação. Todos os testes foram realizados no mesmo dia, em aproximadamente uma hora de avaliação.

Para a avaliação do equilíbrio estático foi aplicado o teste de apoio unipodal 
$(\mathrm{TAU})^{25} \mathrm{e}$, para o equilíbrio dinâmico, o teste de velocidade máxima de andar $(\mathrm{VMA})^{26}$. Ambos foram efetuados com a presença de avaliadores treinados próximos às participantes, para evitar quedas e garantir sua segurança.

Para o TAU, a participante deveria posicionar-se em pé, descalça, com uma das pernas elevadas (joelho flexionado), com as mãos na cintura e olhar fixo em um ponto a dois metros de distância na altura dos olhos. Foi cronometrado o tempo que a participante se mantinha nessa posição, com limite máximo de 30 segundos. Para familiarização com o teste foram permitidas tentativas iniciais até a participante manter-se na posição. Foram executadas três tentativas para cada membro inferior, alternando-se os lados e calculando a média dos tempos em segundos ${ }^{25}$.

No VMA foi cronometrado o tempo que a participante percorreu andando descalça, com a maior velocidade possível, porém sem correr, ao longo de um corredor de 3,33 metros de comprimento por 33,3 centímetros de largura. O teste foi desconsiderado quando a participante pisava fora do corredor demarcado. Foram realizadas três tentativas e calculada a média em segundos e centésimos de segundo ${ }^{26}$.

$\mathrm{Na}$ análise estatística foram utilizados o coeficiente de correlação de Spearman e o teste não-paramétrico de MannWhitney, adotando-se nível de significância fixado em $5 \%(\alpha=0,05)$. As correlações foram consideradas fortes quando $r \geq 0,70$, moderadas se $0,70<r>0,30$ e fracas quando $r \leq 0,30$. A análise foi feita com auxílio do programa SPSS (v.10.0 for Windows).

\section{RESULTADOS}

A amostra foi constituída por 80 mulheres distribuídas em grupo nãoobeso $(n=45)$ e grupo obeso $(n=35)$. A média de idade no grupo não-obeso foi 64 anos, enquanto no grupo obeso foi 63 anos; não foi verificada diferença na média de idade dos grupos (Tabela 1). Como os grupos foram formados segundo o valor de IMC das participantes (não obeso, $<25 \mathrm{~kg} / \mathrm{m}^{2}$ e obeso, $=30,0 \mathrm{~kg} / \mathrm{m}^{2}$ ) a diferença entre ambos quanto ao IMC

Tabela 1 Idade, índice de massa corporal (IMC), gordura corporal (estimada por bioimpedância), resultados obtidos nos testes de equilíbrio estático (TAU) e dinâmico (marcha) e valor de p da comparação entre os grupos de voluntárias não-obesas $(n=45)$ e obesas $(n=35)$

\begin{tabular}{|c|c|c|c|c|c|c|c|}
\hline & Grupo & Mediana & Média & $\mathrm{dp}$ & Mínimo & Máximo & $p$ \\
\hline \multirow{2}{*}{ Idade (anos) } & Não-obesas & 62,00 & 63,91 & 8,38 & 50,00 & 88.00 & \multirow{2}{*}{0,648} \\
\hline & Obesas & 61,00 & 62,91 & 7,30 & 50,00 & 79.00 & \\
\hline \multirow{2}{*}{ IMC $\left(\mathrm{kg} / \mathrm{m}^{2}\right)$} & Não-obesas & 23,54 & 23,12 & 1,39 & 19,86 & 25,04 & \multirow{2}{*}{$<0,01$} \\
\hline & Obesas & 32,93 & 33,71 & 2,89 & 30,12 & 39,93 & \\
\hline \multirow{2}{*}{$\begin{array}{l}\text { Bioimpe- } \\
\text { dância (\%) }\end{array}$} & Não-obesas & 33,7 & 34,16 & 4,93 & 20,1 & 46 & \multirow{2}{*}{$<0,01$} \\
\hline & Obesas & 42,5 & 43,04 & 2,71 & 37,1 & 47,8 & \\
\hline \multirow{2}{*}{$\begin{array}{l}\text { Bioimpe- } \\
\text { dância (kg) }\end{array}$} & Não-obesas & 19 & 19,02 & 2,92 & 11,9 & 23,8 & \multirow{2}{*}{$<0,01$} \\
\hline & Obesas & 33,6 & 35,05 & 5,49 & 26,6 & & \\
\hline \multirow{2}{*}{ TAU D (s) } & Não-obesas & 30,00 & 25,58 & 7,78 & 2,00 & 30,00 & \multirow{2}{*}{$<0,01$} \\
\hline & Obesas & 21,00 & 19,00 & 10,14 & 2,00 & 30,00 & \\
\hline \multirow{2}{*}{$\mathrm{TAU} \mathrm{E}(\mathrm{s})$} & Não-obesas & 30,00 & 24,91 & 8,46 & 2,00 & 30,00 & \multirow{2}{*}{$<0,01$} \\
\hline & Obesas & 17,00 & 17,54 & 9,23 & 2,00 & 30,00 & \\
\hline \multirow{2}{*}{ Marcha (s) } & Não-obesas & 1,90 & 1,98 & 0,40 & 1,44 & 3,87 & \multirow{2}{*}{0,027} \\
\hline & Obesas & 2,05 & 2,08 & 0,32 & 1,64 & 3,35 & \\
\hline
\end{tabular}

$\mathrm{TAU}=$ tempo de apoio unipodal; $\mathrm{D}=$ direito; $\mathrm{E}=$ esquerdo; Marcha = tempo gasto para percorrer 3,33m no teste de velocidade máxima de andar

foi, como esperado, significante $(p<0,01$ - Tabela 1). Em ambos os grupos foi encontrada alta correlação entre a variável IMC e a bioimpedância em \% e em kg (respectivamente $r=0,78 ; p<0,01$ e $r=0,92 ; p<0,01)$.

No grupo obeso, foram capazes de atingir o tempo máximo de 30 segundos $22,9 \%$ das participantes no TAU direito e $17,1 \%$ no esquerdo. No grupo nãoobeso, $57,8 \%$ e $55,6 \%$ das participantes atingiram esse tempo no TAU direito e esquerdo, respectivamente. Como mostra o Gráfico 1, o grupo não-obeso apresentou melhor desempenho no TAU em relação ao grupo obeso, com diferença estatística significativa $(p<0,01)$.

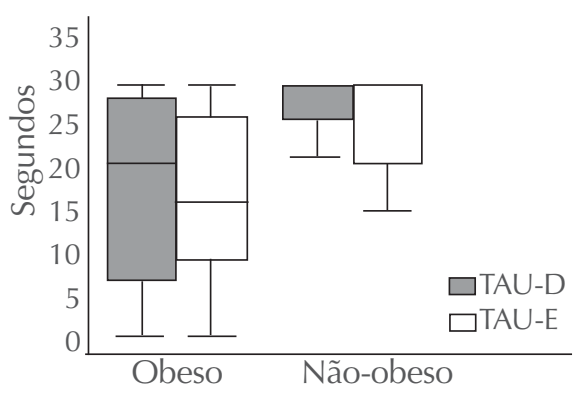

Gráfico 1 Tempo (s) médio de apoio no teste de apoio unipodal direito (TAU-D) e esquerdo (TAU-E) dos grupos obeso $(n=35)$ e não-obeso $(n=45)$
O Gráfico 2 mostra que o grupo nãoobeso foi mais rápido no teste VMA em relação ao grupo obeso, com diferença significativa $(p=0,027)$. O grupo nãoobeso completou o percurso com velocidade média de $2,31 \mathrm{~m} / \mathrm{s}$ e o grupo obeso, com 2,03 m/s.

Quando os valores de bioimpedância foram testados com as variáveis de equilíbrio do grupo não-obeso, foram encontradas correlações moderadas tanto com a medida em \% ( $r=0,69 \mathrm{com}$ TAU direito, $r=0,62$ com TAU esquerdo, $r=0,60$ com VMA, $p<0,01$ em todas), quanto em $\mathrm{kg}(r=0,47$ com TAU direito, $r=0,41$ com TAU esquerdo, $p<0,01$ em ambas, e com VMA, $r=0,31 p<0,05)$. Nos

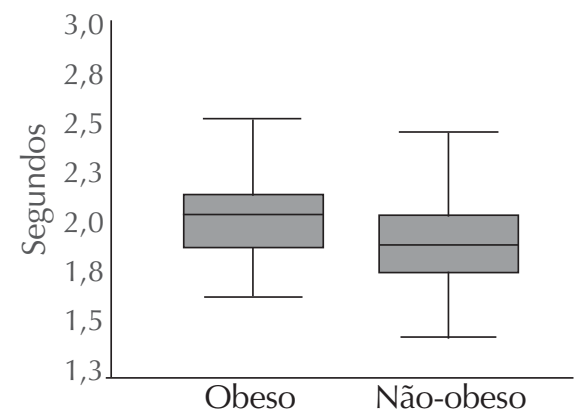

Gráfico 2 Tempo (s) médio gasto para percorrer 3,33 $\mathrm{m}$ no teste de velocidade máxima de andar dos grupos obeso $(n=35)$ e não-obeso $(n=45)$ 
valores obtidos pelo grupo obeso, foi encontrada correlação moderada entre o VMA e a bioimpedância em \% $(r=0,48$; $p<0,01)$, mas não em $\mathrm{kg}(r=0,07$; $p>0,05)$; e não foi verificada correlação entre as medidas de equilíbrio estático e a bioimpedância em $\%(p>0,05)$ nem em kg.

\section{DISCUSSÃO}

A obesidade é um fator associado ao risco de quedas em idosos, tendo impacto negativo no equilíbrio e oscilação postural, além de aumentar o risco de limitações funcionais ${ }^{27}$. Nos obesos observa-se uma alteração do centro de gravidade ${ }^{28}$ que, associada a uma provável redução da força muscular decorrente do envelhecimento, ocasiona maior dificuldade na manutenção do equilíbrio tanto estático quanto dinâmico. Esses dados corroboram os resultados encontrados em ambos os testes de equilíbrio aplicados neste estudo.

O presente estudo verificou que no teste de equilíbrio estático (TAU) o desempenho do grupo não-obeso foi superior ao do grupo obeso. Goulding et al. ${ }^{29}$ compararam o equilíbrio de jovens obesos e não-obesos, não encontrando diferenças no equilíbrio com base de sustentação bipodal; contudo, no equilíbrio unipodal houve alteração significativa pela redução da base de suporte. A quantidade de gordura corporal e a maior dimensão corporal podem ser fatores que dificultam o posicionamento das pernas e a manutenção do equilíbrio no TAU ${ }^{13}$. Greve et al. ${ }^{28}$ observaram correlação entre altos valores de IMC e instabilidade postural em suporte unipodal.

Quanto ao teste de equilíbrio dinâmico, o grupo obeso apresentou maior dificuldade na manutenção do equilíbrio, indicado pelos maiores valores de tempo de percurso e menor velocidade quando comparado ao grupo não-obeso. A média da velocidade de mulheres com aproximadamente 30 anos é de $2,3 \mathrm{~m} / \mathrm{s}^{30}$, velocidade que no presente estudo foi semelhante à encontrada no grupo nãoobeso; já para as idades entre 60 e 70 anos, Hausdorff \& Alexander ${ }^{30}$ observaram que a média declina para $1,7 \mathrm{~m} / \mathrm{s}$, velocidade inferior à encontrada no grupo obeso neste estudo $(2,03 \mathrm{~m} / \mathrm{s})$. A velocidade da marcha no grupo nãoobeso está superior ao esperado para a idade avaliada, visto que a idade mínima da amostra foi 50 anos, enquanto o grupo obeso apresentou velocidades semelhantes ao esperado para a idade avaliada. Obesos possuem aumento da base de apoio durante a marcha para compensar a mudança do centro de gravidade $^{31}$. Os resultados do VMA podem ter sido influenciados pela limitação da base exigida pelo teste (uma faixa de pouco mais de $30 \mathrm{~cm}$ de largura).

Os resultados deste estudo concordam com os de Ferrucci et al. ${ }^{12}$, Barbosa et al..$^{13}$ e Rebelatto et al. ${ }^{18}$, que observaram associação entre equilíbrio e IMC em idosos. Com o avanço da idade, ocorre redução da força muscular, especialmente dos membros inferiores ${ }^{32}$. O processo de envelhecimento acarreta naturalmente uma redução na força, que pode ser acentuada com a obesidade. Obesos apresentam alterações metabólicas que reduzem sua capacidade de hipertrofia muscular ${ }^{16}$. Alterações da força limitam diretamente a manutenção do equilíbrio, pois o sistema osteomuscular é o componente efetor do sistema de controle postural ${ }^{33}$.

Teasdale et al. ${ }^{34}$ observaram que a perda de peso em homens obesos ocasionou melhora do controle postural. Os obesos apresentam aumento na demanda de esforço motor e maior dificuldade de regular o equilíbrio sobre perturbações externas $^{34,35}$. Além disso, a pelve da mulher é mais larga e em obesas há maior quantidade de massa corporal e de gordura localizada nessa região, influenciando diretamente a postura corporal $^{31}$.

Estudos demonstram que em idosos há correlação positiva entre as variáveis IMC e gordura corporal estimada por bioimpedância ${ }^{4,36}$. Esses dados estão de acordo com os resultados deste estudo. No entanto, uma limitação deste estudo é que, no teste de bioimpedância, foram desconsiderados fatores importantes para sua realização de forma replicável, como não ter ingerido ou bebido nas últimas 4 horas, não ter feito exercício físico nas últimas 12 horas, ter urinado 30 minutos antes do teste; não ter consumido álcool nas últimas 48 horas, não ter tomado diuréticos nos últimos 7 dias - e, também, recomenda-se não avaliar mulheres com retenção hídrica devido ao ciclo menstrual. Não foi possível observar esses cuidados ao testar a amostra. Além disso, a maioria das equações preditivas de gordura em idosos não apresentam validação cruzada, limitando sua aplicabilidade. Segundo Gurgel ${ }^{37}$, a bioimpedância manual apresenta uma tendência de aumento nos valores médios com o avançar da idade. Outros estudos concluem que as fórmulas apresentadas para o cálculo da porcentagem de gordura subestimam a massa gorda, já que foram desenvolvidas para utilização em população jovem ${ }^{38} \mathrm{e}$ uma das alterações decorrentes do processo de envelhecimento é a substituição da massa magra por tecido adipo$\mathrm{so}^{38}$. Os pontos de corte de percentual de gordura para mulheres de 56 a 65 anos são: excelente (18 a 22\%), bom (24 a 26\%), acima da média (27 a 29\%), média (30 a 32\%), abaixo da média (33 a $35 \%$ ), ruim (36 a $38 \%$ ) e muito ruim (39 a $49 \%)^{39}$. Considerando esses pontos de corte, observa-se que o grupo não-obeso apresentava percentagem de gordura corporal abaixo da média $(34,16 \%)$ e o grupo obeso, na faixa muito ruim (43,04\%).

Foi verificada correlação moderada entre as variáveis de equilíbrio e a bioimpedância no grupo não-obeso, mas não no grupo obeso. Em mulheres obesas a manutenção do equilíbrio postural é mais difícil, principalmente durante a marcha, muito embora a base de apoio seja proporcional à morfologia estrutural de cada sujeito. Provavelmente a massa corporal elevada com base de apoio estreita causam uma desvantagem mecânica maior do que a quantidade total de gordura na composição corporal.

Indivíduos obesos são freqüentemente sedentários, havendo correlação inversa entre IMC e nível de atividade física $^{16}$. As limitações deste estudo incluem a falta de controle de fatores como nível de atividade física, quantidade de quedas e força muscular, tendo em vista a influência destes na avaliação do equilíbrio corporal. São necessários outros estudos para elucidar quais componentes do sistema de controle postural são afetados pela obesidade. 


\section{CONCLUSÃO}

Os resultados deste estudo evidenciam que obesas a partir da meiaidade apresentam maior deficit de equilíbrio estático e dinâmico quando comparadas às não obesas, sugerindo que, embora o envelhecimento acarrete degeneração dos sistemas de controle postural, a obesidade é um fator agravante. Apesar das limitações, este estudo guarda importância de natureza clínica, já que pacientes obesos são cada vez mais comuns nos centros de reabilita- ção. A identificação da obesidade por meio do IMC é um método fácil e rápido, que pode fazer parte da rotina de avaliação da fisioterapia para que o enfoque terapêutico leve em consideração os riscos que essa condição acarreta.

\section{REFERÊNCIAS}

1 Lacourt MX, Marini LL. Decréscimo da função muscular decorrente do envelhecimento e a influência na qualidade de vida do idoso: uma revisão de literatura. RBCEH. 2006;3(1):114-21.

2 Villareal DT, Banks M, Siener C, Sinacore DR, Klein S. Physical frailty and body composition in obese elderly men and women. Obes Res. 2004;12(6):913-20.

$3 \mathrm{WHO}$ - World Health Organization. Physical status: the use and interpretation of anthropometry. Geneva: WHO; 1995. (WHO Technical Report Series 854).

4 Cabrera MAS, Jacob Filho W. Obesidade em idosos: prevalência, distribuição e associação com hábitos e comorbidades. Arq Bras Endocrinol Metabol. 2001;45(5):494-501.

5 Rebelatto JR E Castro AP. Efeito do programa de revitalização de adultos sobre a ocorrência de quedas dos participantes. Rev Bras Fisioter. 2007;11(5):383-9.

6 Abrantes MM, Lamounier JA, Colosimo EA. Prevalência de sobrepeso e obesidade nas regiões Nordeste e Sudeste do Brasil. Rev Assoc Med Bras. 2003;49(2):162-6.

7 Aikawa AC, Braccialli LMP, Padula RS. Efeito das alterações posturais e de equilíbrio estático nas quedas de idosos institucionalizados. Rev Cienc Med. 2006;15(3):189-96.

8 Chandler JM. Equilíbrio e Quedas no Idoso: Questões sobre a avaliação e o tratamento. In: Guccione C. Fisioterapia geriátrica. Rio de Janeiro: Guanabara Koogan; 2002. p.265-77.

9 Mille ML, Mouchnino L. Are human anticipatory postural adjustments affected by a modification of the initial position of the center of gravity? Neurosci Lett 1998;242:61-4.

10 Damaso A. Obesidade. Rio de Janeiro: Medsi; 2003.

11 Corbeil P, Simoneau M, Rancourt D. Increased risk for falling associated with obesity: mathematical modeling of postural control. IEEE Trans Neural Syst Rehabil Eng, 2001;9:126-36.

12 Ferrucci L, Penninx BW, Leveille SG, Corti MC, Pahor M, Wallace R, et al. Characteristics of nondisabled older persons who perform poorly in objective tests of lower extremity function. J Am Geriatr Soc. 2000;48(9):1102-10.
13 Barbosa AR, Souza JMP, Lebrão ML, Marucci MFN. Estado nutricional e desempenho motor de idosos de São Paulo. Rev Assoc Med Bras. 2007;53(1):75-9.

14 McGraw B, McClenaghan BA, Williams HG, Dickerson J, Ward DS. Gait and postural stability in obese and nonobese prepubertal boys. Arch Phys Med Rehabil. 2000;81:484-9.

15 Santana LA, Gonçalves CA. A influência da visão no equilíbrio estático de crianças pré-púberes obesas. In: 9ํo Congresso Brasileiro de Biomecânica, 2001, Porto Alegre. Anais. São Paulo: SBB; 2001. p.110-3.

16 Fjeldstad C, Fjeldstad AS, Acree LS, Nickel KJ, Gardner AW. The influence of obesity on falls and quality of life. Dyn Med [periódico on-line]. 2008;7:4. Disponível em: http://www.dynamic-med.com/content/7/1/4.

17 Bruce DG, Devine A, Prince RL. Recreational physical activity in healthy older women: the importance of fear of falling. J Am Geriatr Soc. 2002;50:84-9.

18 Rebelatto JR, Castro AP, Sako FK, Aurichio TR. Equilíbrio estático e dinâmico em indivíduos senescentes e o índice de massa corporal. Fisioter Mov. 2008;21(3):69-75.

19 Anjos LA. Índice de massa corporal como indicador de estado nutricional de adultos: revisão de literatura. Rev Saude Publica. 1992;26(6):431-6.

20 Sampaio LR, Figueiredo VC. Correlação entre o índice de massa corporal e os indicadores antropométricos de distribuição de gordura corporal em adultos e idosos. Rev Nutr. 2005;18(1):53-61.

21 WHO - World Health Organization. Obesity: preventing and managing the global epidemic; report of a WHO consultation. Geneva; 2000. (WHO Technical Report Series 854).

22 Buscariolo FF, Catalani MC, Dias LCGD, Navarro AM. Comparação entre os métodos de bioimpedância e antropometria para avaliação da gordura corporal em atletas do time de futebol feminino de Botucatu/SP. Rev Simbio-Logias. 2008;1(1):122-9.

23 Kushner RF. Bioelectrical impedance analysis: a review of principles and applications. J Am Coll Nutr. 1992;11(2):199-209. 


\section{Referências (cont.)}

24 Chumlea WC, Guo SS, Kuczmarski RJ, Vellas B. Bioelectrical and anthropometric assessments and reference data in the elderly. J Nutr. 1993;123:449-53.

25 Bohannon RW. One-legged balance test times. Percept Mot Skills. 1994;78(3):801-2.

26 Bohannon RW, Andrews AW, Thomas MW. Walking speed: reference values and correlates for older adults. J Orthop Sports Phys Ther. 1996;24:86-90.

27 McTigue KM, Hess R, Ziouras J. Obesity in older adults: a systematic review of the evidence for diagnosis and treatment. Obesity. 2006;14(9):1485-97.

28 Greve J, Alonso A, Bordini ACPG, Camanho GL. Correlation between body mass index and postural balance. Clinics. 2007;62(6):717-20.

29 Goulding A, Jones IE, Taylor RW, Piggot, JM, Taylor D. Dynamic and static tests of balance and postural sway in boys: effects of previous wrist bone fractures and high adiposity. Gait Posture. 2003;17:136-41.

30 Hausdorff JM, Alexander NB. Gait disorders: evaluation and management. Boca Raton: Taylor \& Francis; 2005.

31 Bankoff ADP, Zamai CA, Schimdt A, Ciol P, Barros DD. Estudo das alterações morfológicas do sistema locomotor: postura corporal x obesidade. Rev Educ Fisica UEM. 2003;14(2):41-8.

32 Graf A, Judge JO, Õunpuu S, Thelen DG. The effect of walking speed on lower-extremity jointpowers among elderly adults who exhibit low physical perfomance. Arch Phys Med Rehabil. 2005;86:2177-83.
33 Degens $\mathrm{H}$, Erskine RM, Morse Cl. Disproportionate changes in skeletal muscle strength and size with resistance training and ageing. J Musculoskelet Neuronal Interact. 2009;9(3):123-9.

34 Teasdale N, Hue O, Marcotte J, Berrigan F, Simoneau M, Doré J, et al. Reducing weight increases postural stability in obese and morbid obese men. Int J Obes (Lond). 2007;31(1):153-60.

35 Wearing SC, Henning EM, Byrne NM, Steele JR, Hills AP. The biomechanics of restricted movement in adult obesity. Obes Rev. 2006;7:13-24.

36 Justino SR, Souza MH, Simeone G, Gomide PIC, Malafaia O. Correlação entre medidas antropométricas e massa corporal gorda avaliado por bioimpedância em mulheres idosas não-institucionalizadas. Rev Med Parana, 2005;63(2):18-21

37 Gurgel JL. Avaliação do perfil antropométrico dos idosos de Porto Alegre, RS: a influência da metodologia na determinação da composição corporal [tese]. Porto Alegre: Pontifícia Universidade Católica do Rio Grande do Sul; 2008.

38 Santos LJM. Indicadores de saúde em mulheres acima de 50 anos de idade participantes de um programa de atividades físicas no complexo da Maré, RJ. [dissertação]. Campinas: Universidade Estadual de Campinas; 2004.

39 Pollock ML, Wilmore JH. Exercícios na saúde e na doença: avaliação e prescrição para prevenção e reabilitação. São Paulo: MEDSI; 1993. 